\title{
Medical Therapy Update in Crohn's Disease
}

\author{
Andrew DuPont ${ }^{1,2}$ and Atilla Ertan ${ }^{1,2 *}$ \\ ${ }^{1}$ Ertan Digestive Disease Center-Inflammatory Bowel Disease Center, USA \\ ${ }^{2}$ University of Texas Health Science Center Gastroenterology, Hepatology and Nutrition Division and \\ Memorial Hermann Hospital, USA
}

Received: February 11, 2014; Accepted: February 12, 2014; Published: February 13, 2014

*Corresponding author: Atilla Ertan, The UTH-MHH Ertan Digestive Disease Center and Gastroenterology Center of Excellence, 6400 Fannin, Suite 1400, Houston, Texas 77030, USA, Tel: 713-704-5928, 713-704-3450; Fax: 713-704-3485; E-mail: atilla.ertan@uth.tmc.edu

\section{Editorial}

Crohn's disease (CD) is a chronic inflammatory bowel disease that is suspected to result from a dysregulated immune system in genetically predisposed individuals, which can lead to abdominal pain, diarrhea, malnutrition and extra- intestinal manifestations. Intestinal inflammation and subsequent symptoms tend to recur over time and can lead to other complications, such as fistulas, abscesses, and strictures. CD is a chronic progressive condition, with most patients developing penetrating and/or stricturing complications over time.

Previously, only limited treatment options existed; however, over the past 30 years progress has been made regarding management of $\mathrm{CD}$. The goals of treatment include: healing inflammation (mucosal healing; and ideally transmural healing), preventing recurrent inflammation and symptoms, reducing and eliminating the use of steroids, decreasing hospitalizations and surgical intervention, preventing complications, improving quality of the life and alternate the natural course of CD.

Initially, treatment for CD consisted of corticosteroids, other anti- inflammatory medications (5-aminosalicylates), and often times surgery. Corticosteroids can reduce inflammation anywhere in the body, but it is not effective for long-term treatment of patients with $\mathrm{CD}$ and is associated with many significant side effects.

Other treatments have been developed to directly suppress the immune response, rather than only treating inflammation, which is the result of the abnormal immune response. These medications include immunomodulators (azathioprine, 6-mercaptopurine, and methotrexate) and more recently biologic agents (infliximab, adalimumab, certolizumab, and natalizumab). It is generally recommended patients with $\mathrm{CD}$ be treated with the combination of immunomodulator therapy and a biologic agent to increase the likelihood of steroid-free induction and longterm remission [1,2]. According to our experience and general concensus, patients with CD who should be considered for early biologic therapy include: young age at disease onset, steroidrefractory disease, deep ulceration or fistulizing disease, multiple antibodies to intestinal flora, perianal disease, and extensive disease in the upper small intestine.
Infliximab (Remicade) was the first biologic agent approved for the management of $C D$. It is an antibody that binds to the tumor necrosis factor (TNF), which blocks its activity and leads to decreased inflammation. Infliximab consists of a partial mouse antibody that is given as an intravenous infusion over a period of at least 2-3 hours. It is initially infused 3 times over a 6 week period and if there is significant improvement, every 2 months for maintenance therapy $[1,2]$. Infliximab is generally well tolerated; however, rarely it can be associated with infusion reactions. Because TNF is an important component of the immune system, infliximab and other biologic medications affect the body's ability to fight infections, so patients should be checked for tuberculosis and hepatitis B prior to starting these medications. It is also important to avoid live vaccines while taking these medications [1].

Adalimumab (Humira) is another anti-TNF agent similar to infliximab, which decreases inflammation by blocking TNF; however, adalimumab consists of a fully humanized anti-TNF antibody, and it is administered by subcutaneous injection, rather than an infusion. After the loading dose is given in 2 doses over a 2 week period, it is administered every other week for maintenance therapy [3]. It is also generally well- tolerated with the most common side effect being skin reactions at the site of the injection that may cause itching, redness, and swelling. Adalimumab has been shown to have similar effectiveness and safety when compared to infliximab for inducing and maintaining remission in patients with $\mathrm{CD}$. It has also been shown to be effective for some patients who have failed or cannot tolerate infliximab [1]

Certolizumab pegol (Cimzia) is a pegylated humanized antibody fragment that also acts by blocking the activity of TNF. It was approved for use in the US in 2008 for the treatment of moderate to severe $\mathrm{CD}$ in patients who do not respond sufficiently or adequately to standard medical therapy. Certolizumab is a subcutaneous injection, similar to adalimumab, and is given twice over a 2 week period then once a month for maintenance therapy. It has been shown to be effective in inducing and maintaining remission in Crohn's disease with similar adverse events as seen with Infiliximab and Adalimumab [4]. It has also been shown to be effective for some patients who have failed or cannot tolerate infliximab. 
Natalizumab (Tysabri) is a humanized monoclonal antibody to alpha-4 integrin, which acts by affecting lymphocytic trafficking. It has been approved for moderate to severe CD that has not responded to other treatment. It is given as an IV infusion over 1 hour once a month [5]. Natalizumab should not be used with other immunosuppressive medications. Because it has been associated with a rare, but serious, brain infection (progressive multifocal leukoencephalopathy, PML), it is only available through a special restricted distribution program. Vedolizumab, a newer biologic agent, also affects lymphocytic trafficking. According to well- designed studies, this agent induces a gutselective blockade of alpha- 4 beta- 7 integrin which appears to eliminate the risk of PML with similar efficacy when compared to other commercially available biologics for the treatment of patients with $\mathrm{CD}[6,7]$. Vedolizumab is expected to be approved by the FDA for the treatment of patients with CD within 2014 and appears to be another promising new biologic agent.

These agents have never been directly compared headto- head, and the decision to start one or another should be individualized [1]. Biologic therapy with these commercially available agents leads to clinical remission in approximately $30 \%$ and clinical response in $50 \%$ of patients with active CD [1-8].Among those CD patients who respond initially to antiTNF agents, up to $30-40 \%$ will lose response over time [1-8]. Therefore, extensive investigations with newer agents with different mechanisms are needed and are in progress (Table 1).

Interleukin (IL)-12 and IL-23 have been shown to play an important role in $\mathrm{T}$ helper cell and innate lymphocyte cell differentiation and expansion. Ustekinumab (Stelara), a fully humanized IgG1 monoclonal antibody to the p40 subunit shared by IL-12 and IL-23, has emerged as a very promising treatment option, especially in complicated CD patients who are refractory to other biologics $[9,10]$. Other potential agents currently in development are shown in Table 1, which includes newer oral agents [11-15]. Fecal microbiota transplantation has also been reported to be successful in small case series and case reports for the treatment of $\mathrm{CD}$; however, more research is needed in this area.

\section{Acknowledgement}

Supported by Ertan Research and Education Foundation.

Table 1: New agents in development for the treatment of Crohn's Disease.

\begin{tabular}{|c|c|c|}
\hline Name & Major Effect & Product \\
\hline Etrolizumab & $\begin{array}{c}\text { Antibody to beta-7 of } \\
\text { integrins }\end{array}$ & $\begin{array}{c}\text { Humanized GI } \\
\text { specific MCA }\end{array}$ \\
\hline Ustekinumab & Anti-IL- 12/23 P40 & Human IgG1 MCA \\
\hline Tocilizumab & Anti-IL-6 & Humanized MCA \\
\hline Secukinumab & Anti-IL-17 & Humanized MCA \\
\hline Tofacitinib* & Janus kinase inhibitor & Immunomodulator \\
\hline Fingolimod* & $\begin{array}{c}\text { Sphingosine 1PR1 } \\
\text { modulator }\end{array}$ & $\begin{array}{c}\text { Lymphocyte receptor } \\
\text { agonist }\end{array}$ \\
\hline HMPL-004* & Herbal mixture & Anti-inflammatory \\
\hline GSK1605786* & CCR9 antagonist & $\begin{array}{c}\text { GI specific } \\
\text { immunomodulator }\end{array}$ \\
\hline
\end{tabular}

*Oral Agents

\section{References}

1. Feagan BG, Lemann M, Befrits R, Connell W, D'Haens G, et al. (2012) Recommendations for the treatment of Crohn's disease with tumor necrosis factor antagonists: an expert consensus report. Inflamm Bowel Dis 18(1): $152-160$

2. Colombel JF, Sanborn WJ, Reinisch W, Mantzaris GJ, Kornbluth A, et al. (2010) Infliximab, azathioprine, or combination therapy for Crohn's disease. N Engl J Med 362(15): 1383-1395.

3. Rutgeerts P, Van Assche G, Sanborn WJ, Wolf DC, Geboes K, et al. (2012) Adalimumab induces and maintains mucosal healing in patients with Crohn's disease: data from the EXTEND trial. Gastroenterology 142(5): 1102-1111.

4. Colombel JF, Lemann M, Bouhnik Y, Dewit O, Dupas JL, et al. (2010) Endoscopic mucosal improvement in patients with active Crohn's disease treated with Certolizumab Pegol: week 10 and 54 results of the music trials. AGA Gastroenterology 138(5): S-166.

5. Van AG, Van RM, Sciot R, Dubois B, Vermeire S, et al. (2005) Progressive multifocal leukoencephalopathy after natalizumab therapy for Crohn's disease. N Engl J Med 353(4): 362-368.

6. Danese S, Colombel JF, Reinisch W, Rutgeerts PJ (2011) Review article: infliximab for Crohn's disease treatment- shifting therapeutic strategies after 10 years of clinical experience. Aliment Pharmacol Ther 33(8): 857869.

7. Sandborn WJ, Feagan BG, Rutgeerts P, Hanauer S, Colombel JF, et al. (2013) Vedolizumab as induction and maintenance therapy for Crohn's disease. N Engl J Med 369(8): 711-721.

8. Lobaton T, Vermeire S, Van AG, Rutgeerts P (2014) Review article: anti- adhesion therapies for inflammatory bowel disease. Alimentary Pharmacology and Therapeutics DOI: 10, 1111/apt. 12639.

9. Sandborn WJ, Gasink C, Gao LL, Blank MA, Johanns J, et al. (2012) Ustekinumab induction and maintenance therapy in refractory Crohn's disease. N Engl J Med 367(16): 1519-1528.

10. Bouguen G, Chevaux JB, Peyrin BL (2011) Recent advances in cytokines: therapeutic applications for inflammatory bowel diseases. World J Gastroenterol 17(5): 547-556.

11. Vuitton 1, Koch S, Peyrin BL (2013) Janus kinase inhibition with tofacitinib: changing the face of inflammatory bowel disease treatment. Curr Drug Targets 14(12): 1385-1391.

12. Perrier C, Rutgeerts P (2012) New drug therapies on the horizon for IBD. Dig Dis 30: 100-105.

13. Danese S (2012) New therapies for inflammatory bowel disease: from the bench to the bedside. Gut 61(6): 918-932.

14. Rietdjik ST, D’Haens GR (2013) Recent developments in the treatment of inflammatory bowel disease. J Dig Dis 14(6): 282-287.

15. Neurath MF (2014) New targets for mucosal healing and therapy in inflammatory bowel disease. Mucosal Immunology 7: 6-19. 